\title{
FOCO NO CLIENTE EM TIMES DE DESENVOLVIMENTO DE SOFTWARE: UM ESTUDO QUANTITATIVO
}

\author{
CUSTOMER FOCUS IN SOFTWARE DEVELOPMENT TEAM: \section{A QUANTITATIVE STUDY}

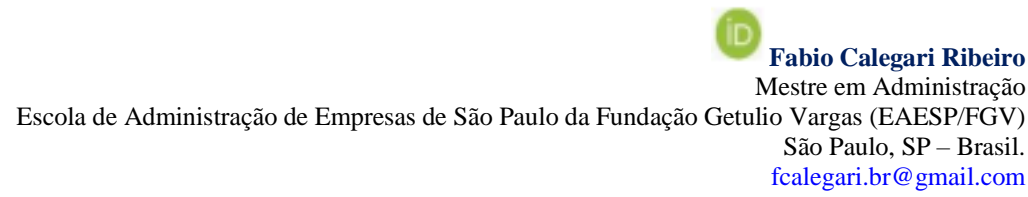

Cláudio Luís Carvalho Larieira Doutor em Administração Escola de Administração de Empresas de São Paulo da Fundação Getulio Vargas (EAESP/FGV) São Paulo, SP - Brasil claudio.larieira@fgv.br

Adilson Carlos Yoshikun Doutor em Administração Universidade Presbiteriana Mackenzie São Paulo, SP - Brasil. adilson.yoshikuni@mackenzie.br

Ivanir Costa
Doutor em Engenharia de Produção
Universidade Nove de Julho
São Paulo, SP - Brasil.
ivanirc@uni9.pro.br

\section{Resumo}

No início dos anos 2000 o Manifesto Ágil consolidou as práticas ágeis como contraponto ao modelo tradicional de gestão de projetos, considerando entre outras, a colaboração com o cliente e a aceitação às mudanças durante o processo de desenvolvimento de software como princípios básicos. Muitas empresas têm implementado métodos ágeis por influências externas e internas à organização, mas não capturam os principais benefícios da filosofia e continuam focando em eficiência ao invés de eficácia, através da perspectiva utilizada nos modelos tradicionais de gestão de projetos, levando à questão sobre quais fatores são mais importantes ao implementar métodos ágeis com foco no cliente. O objetivo desta pesquisa é avaliar os fatores que influenciam os times ágeis de desenvolvimento na obtenção de foco no cliente. Para avaliar estes fatores foi elaborado um questionário, que foi respondido por profissionais das áreas de TI e negócios que tem ou tiveram contato com métodos ágeis, no Brasil. Os resultados demonstram entre os fatores coleta e entendimento de requisitos, coleta e uso de informações do cliente, recebimento e utilização de feedback do cliente e relacionamento com o cliente quais possuem maior e menor efeito na mensuração do foco no cliente em times ágeis, contribuindo com os profissionais que implementam métodos ágeis em suas organizações para atingir um dos principais objetivos destes métodos, que é a satisfação do cliente.

Palavras-chave: Métodos ágeis. Foco no cliente. Satisfação do cliente. Práticas ágeis. Times ágeis

Abstract

In the early 2000s, the Agile Manifesto consolidated agile practices as a counterpoint to the project management traditional model, considering among others, collaboration with the customer and acceptance of changes during the software development process as basic principles. Many companies adopted agile methods due to external and internal influences in your organization but fail to capture the main benefits of the agile philosophy and continue to focus on efficiency rather than effectiveness through plan driven methods perspective, leading the question about which factors are most important when implementing agile methods focusing on customer. The objective of this research was evaluated which factors influence on agile teams to obtain customer focus. To evaluate these factors was used online survey shared with IT and business Brazilian professionals that working on agile methods and the results demonstrate among gathering and understanding of customer requirements, collection and utilization of customer information, receiving and utilization of customer feedback and improvement of customer relationships which have greater and lower effects on customer focus in agile teams, contributing with professionals that are implementing agile methods to achieve one of the most important objective from these methods that is the customer satisfaction.

Keywords: Agile methods. Customer focus. Customer satisfaction. Agile practices. Agile teams

\section{Cite como}

American Psychological Association (APA)

Ribeiro, F. C., Larieira, C. L. C., Yoshikuni, A. C., \& Costa, I. (2021, set./dez.). Foco no cliente em times de desenvolvimento de software: um estudo quantitativo. Revista de Gestão e Projetos (GeP), 12(3), 36-59. https://doi.org/10.5585/gep.v12i3.20254 


\section{Introdução}

Um número maior de empresas tem adotado métodos ágeis nos projetos de desenvolvimento de software (Gandomani \& Nafchi, 2016) por influência de clientes, parceiros, mídia, instituições profissionais e da alta gerência (Lee \& Chen, 2019). De acordo com o estudo de Ghani, Bello e Bagiwa (2015), o benefício obtido por quem adota métodos ágeis é a melhoria em aspectos importantes no processo de desenvolvimento de software quando comparados aos métodos tradicionais de gestão de projetos: $62 \%$ dos respondentes afirmam que o prazo de entrega melhorou, $66 \%$ afirmam que o processo de gestão de mudanças também melhorou e $50 \%$ afirmam que o grau de satisfação do cliente aumentou entre $50 \%$ e $90 \%$.

Segundo Gandomani e Nafchi (2016), os principais objetivos ao utilizar métodos ágeis são: aceleração na entrega de produtos, melhor gestão de mudança de prioridades, melhor alinhamento com o negócio, maior comprometimento das pessoas, aumento de produtividade, melhoria na qualidade de software e principalmente a satisfação do cliente. Porém, ao adotar estes métodos sem levar em conta sua cultura, as empresas deixam de obter os resultados esperados (Lee \& Chen, 2019).
Ao avaliar projetos de desenvolvimento de software através de perspectivas relacionadas aos métodos tradicionais de gestão de projetos sem levar em conta o resultado percebido pelo cliente, as empresas podem considerar o resultado destes projetos um sucesso, por serem entregues dentro do prazo e orçamento esperado, mesmo sem entregar valor para o cliente (Collyer, Warren, Hemsley, \& Stevens, 2010).

Para que os métodos ágeis sejam percebidos de maneira positiva é essencial o foco no cliente durante o processo de desenvolvimento de software, seja o cliente interno ou externo à organização, uma vez que o Manifesto Ágil propõe como um de seus princípios a satisfação do cliente (Beck, Cunningham, Hunt, Martin, Thomas, Beedle, \& Sutherland, 2001). A preocupação e consequentemente satisfação do cliente normalmente é dividida em quatro categorias: tempo, qualidade, performance e serviço, e custo (Kaplan \& Norton, 2005).

Empresas com foco no cliente são mais inovadoras e tem melhor desempenho em indicadores financeiros e não financeiros (Grinstein, 2008; Hult \& Ketchen Jr, 2001; Kirca, Bearden, \& Hult, 2011; Kirca, Jayachandran, \& Bearden,, 2005). A orientação para o cliente pode ser 
responsiva ou proativa, utilizando informações disponíveis dos clientes existentes ou descobrindo necessidades não expressadas por eles, respectivamente (Hair Jr, Gabriel, \& Patel, 2014).

O estudo de Lohan, Conboy e Lang (2011) descreve o foco no cliente em times de desenvolvimento ágeis, demonstrando quatro fatores que influenciam o processo de desenvolvimento para obtenção de foco na entrega de valor para o cliente, sendo eles: coleta e entendimento de requisitos, coleta e uso de informações do cliente, recebimento e utilização de feedback do cliente e relacionamento com o cliente.

$\mathrm{Na}$ literatura existente não foram encontrados estudos que abordem comprovação sobre fatores de influência em times de desenvolvimento ágil no Brasil quando se deseja obter foco no cliente, levando à questão de pesquisa sobre quais são estes fatores e seus efeitos em times ágeis no Brasil com base no estudo de Lohan et al. (2011), utilizando uma abordagem quantitativa, através do método PLS-PM (partial least squares path modeling).

Esta pesquisa foi realizada com uso de formulário online, junto a profissionais que tenham ou tiveram contato com métodos ágeis no Brasil, complementando estudos de levantamento de dados (Ghani et al., 2015; Lishner \& Shtub, 2019; Serrador \& Pinto, 2015; Sheffield \& Lemétayer, 2013) sobre a adoção e sucesso de métodos ágeis, contribuindo com a literatura existente ao indicar para os profissionais que implementam estes métodos em suas organizações quais são os fatores e suas respectivas relevâncias no processo de desenvolvimento de software, bem como as principais práticas associadas a estes fatores, ajudando assim a aumentar a satisfação do cliente.

\section{Revisão de literatura}

A revisão de literatura tem como objetivo analisar a literatura existente sobre métodos ágeis, principais motivadores para adoção e como isso influencia o processo de desenvolvimento tendo o cliente como foco. Com este objetivo foram abordados os seguintes aspectos nesta seção: introdução aos métodos ágeis, adoção de métodos ágeis, métricas de produtividade e valor para o cliente, critérios de avaliação na adoção de métodos ágeis e desenvolvimento ágil com foco no cliente.

\subsection{Introdução aos métodos ágeis}

Os princípios da filosofia ágil estão descritos no Manifesto Ágil (Beck et al., 2001), que é a base referencial para os métodos que se consolidaram 
posteriormente. São doze os princípios e

são apresentados na Tabela 1.

Tabela 1.

Princípios do Manifesto Ágil

\section{Princípios}

Nossa maior prioridade é satisfazer o cliente por meio de entrega antecipada e contínua de software valioso. Bem-vindo a mudanças de requisitos, mesmo no final desenvolvimento. Os processos ágeis aproveitam a mudança para a vantagem competitiva do cliente.

Entregar software funcional com frequência, a partir de algumas semanas ou meses, com preferência para a escala de tempo mais curta.

Pessoas de negócio e desenvolvedores devem trabalhar juntos diariamente ao longo do projeto.

Construa projetos em torno de indivíduos motivados. Dê a eles o ambiente e o suporte de que precisam e confie neles para fazer o trabalho.

O método mais eficiente e eficaz de transmitir informações para e dentro de um time de desenvolvimento é uma conversa cara a cara.

O software funcional é a principal medida de progresso.

Processos ágeis promovem o desenvolvimento sustentável. Os patrocinadores, desenvolvedores e usuários devem ser capazes de manter um ritmo constante indefinidamente.

Atenção contínua à excelência técnica e um bom design aumentam a agilidade.

Simplicidade - a arte de maximizar a quantidade de trabalho não feito - é essencial.

As melhores arquiteturas, requisitos e projetos emergem de times auto-organizados.

Em intervalos regulares, o time reflete sobre como se tornar mais eficaz, então sintoniza e ajusta seu

comportamento de acordo

Fonte: Elaborado com base em Beck et al. (2001).

A maior parte das práticas ágeis não são novas e sim consequência de um processo evolutivo na área de desenvolvimento de software, onde os processos são constantemente melhorados com base nos sucessos e fracassos de projetos anteriores (Cohen, Lindvall, \& Costa, 2004). A filosofia ágil prescreve o uso de processo incremental e interativo de desenvolvimento, quebrando o modelo tradicional em pequenos ciclos, com demonstração de software funcionando e coleta de feedback de quem o utilizará, indicando se o produto que está sendo entregue está correto ou não e permitindo correção e mudanças após cada um dos ciclos (Beck, 1999).

Um dos valores declarados dos métodos ágeis é a aceitação às mudanças, sendo descrito como "responder a mudanças mais que seguir um plano" (Beck et al., 2001), o que vai de encontro ao atendimento das necessidades do cliente, que de fato mudam durante o processo de 
desenvolvimento de software, fazendo um contraponto aos métodos tradicionais que prescrevem a execução do plano inicial evitando mudanças (Boehm, 2002). Outro valor do manifesto ágil, que vai de encontro à este estudo é o foco no cliente descrito como "colaboração com o cliente mais que negociação de contratos" (Beck et al., 2001).

\subsection{Adoção de métodos ágeis}

Os métodos plan-driven, também chamados de waterfall, foram tradicionalmente usados por pequenas e grandes empresas nas áreas de desenvolvimento de software (Ghani et al., 2015), mas tem aumentado de maneira significativa o número de empresas que implementam métodos ágeis (Gandomani \& Nafchi, 2016). Ambos os métodos tem seus benefícios e limitações, porém para parte das empresas que implementam métodos ágeis não estão claros os motivos para isso, o que pode tornar a transição mais difícil do que o esperado (Gandomani \& Nafchi, 2016).

As empresas são pressionadas a adotar métodos ágeis por fatores externos e internos buscando aceitação do mercado para atingir o sucesso e sobreviverem (Lee \& Chen, 2019). Um dos fatores externos que gera forte influência na adoção de métodos ágeis é a coerção de clientes, que precisam que seus parceiros adotem novos modelos de desenvolvimento de software que se adequem aos seus ou de seus parceiros (Lee \& Chen, 2019). Outros fatores externos estão relacionados à mídia e ás instituições profissionais que também podem exercer grande influência pois estas instituições dividem as mesmas crenças sobre os objetivos atingidos por determinado padrão, como é o caso dos métodos ágeis (Lee \& Chen, 2019).

Já os fatores internos que influenciam na adoção de métodos ágeis estão relacionados ao desejo da alta gerência e à própria cultura da empresa, na qual as empresas com maior tendência de adoção são mais suscetíveis à flexibilidade e ao arbítrio (Lee \& Chen, 2019).

Os principais benefícios na adoção de métodos ágeis e que deveriam ser levados em consideração na decisão de adotá-los são: aceleração na entrega de produtos, melhor gestão de mudança de prioridades, melhor alinhamento com o negócio, maior comprometimento das pessoas, aumento de produtividade, melhoria na qualidade de software e aumento na satisfação do cliente (Gandomani \& Nafchi, 2016).

Os métodos ágeis geram resultados melhores, com entregas mais assertivas para 
o cliente quando comparados aos tradicionais, em ambientes de maior complexidade ou incerteza (Loch \& Sommer, 2019). Startups se beneficiam dos métodos ágeis, pois trabalham normalmente em um ambiente de grande incerteza por conta de investidores que mudam de atitude repentinamente, por mudanças regulatórias, por mudança no comportamento de seus clientes ou até mesmo pela complexidade de seus produtos que até então não existiam (Loch \& Sommer, 2019).

\subsection{Métricas de produtividade e valor para o cliente}

Nos métodos ágeis as métricas são diferentes das métricas utilizadas nos métodos tradicionais de gestão de projetos, focando na quantidade de requisitos entregues por iteração ou no tempo de espera de cada um destes requisitos, o que são métricas internas de desenvolvimento (Budacu \& Pocatilu, 2018).

Segundo Budacu e Pocatilu (2018) ao utilizar métodos ágeis, ter um sistema de métricas de produtividade em tempo real com foco em métricas internas reduz o tempo de gestão de TI, desde que existam dados históricos consistentes para a comparação de performance entre projetos, mas sugere que estudos futuros poderiam focar em métricas externas como satisfação do cliente, qualidade percebida e receita de produtos, visão compartilhada também por Petersen (2011). Métricas externas que estão diretamente ligadas ao valor de negócio de TI são consonantes com o Manifesto Ágil (Beck et al., 2001), que busca gerar mais valor e de forma mais rápida para o cliente.

Mensurar o valor de TI é menos complexo quando ela é tratada como habilitadora de processos, pois dessa forma é possível medir o aumento da margem de lucro ou a redução de custos implementando ou melhorando sistemas (Ayabakan, Bardhan, \& Zheng, 2017), mas estudos recentes demonstram que também é possível mensurar o valor de estratégias de sistemas de informação nas capacidades dinâmicas e de inovação, que por sua vez influenciam positivamente no desempenho das empresas (Yoshikuni, Galvão, \& Albertin, 2021).

Quando se descreve métricas de produtividade e resultado de TI, apesar da mudança dos métodos tradicionais de gestão de projetos para os métodos ágeis, muitas empresas continuam focando em métricas internas que medem eficiência e não eficácia, quando poderiam estar focando na satisfação de seu cliente e em como o valor de negócio é gerado através de TI. 
2.4 Critérios de avaliação na adoção de métodos ágeis

Métodos ágeis promovem inovação contínua mais do que controle e podem ser considerados um modelo pós-burocrático, que tem três características principais: obsessão pelo cliente, constantes entregas (ainda que pequenas) que geram valor mais rápido e estruturas organizacionais em rede, que colaboram entre si (Denning, 2019).

Os projetos plan-driven são orientados ao plano e seu sucesso é medido dessa forma (Sheffield \& Lemétayer, 2013), enquanto o sucesso de projetos desenvolvidos com métodos ágeis dependem das metas estabelecidas para ele (Serrador \& Pinto, 2015), tais como impacto no cliente, impacto no negócio, valor para a organização ou preparação para o futuro (Sheffield \& Lemétayer, 2013). Quando se pretende medir os resultados gerados pela execução de métodos ágeis, ainda é comum se empregar as mesmas práticas de planejamento e fatores de sucesso do modelo tradicional (Lishner \& Shtub, 2019).

\subsection{Desenvolvimento ágil com foco no cliente}

O primeiro princípio do Manifesto Ágil, conforme demonstrado na Tabela 1, coloca como maior prioridade satisfazer o cliente (Beck et al., 2001) e por isso o estudo utilizado como base para esta pesquisa é o de Foco no Cliente proposto por Lohan et al. (2011). Os métodos ágeis de gestão de projetos ajudam a responder de maneira rápida e extensa os requisitos do cliente (Recker, Holten, Hummel, \& Rosenkranz, 2017) e por isso estão fortemente associados ao estudo de foco no cliente.

Apesar da importância do envolvimento e do foco no cliente em times ágeis, além do fato de que o envolvimento do cliente desde as fases iniciais do projeto ajuda a entender o que é valor para ele, existem várias barreiras para que isso ocorra de forma adequada, sendo as principais: falta de tempo e envolvimento do cliente por falta de confiança, falta de entendimento por parte do cliente, pessoas sem as competências corretas envolvidas nos projetos e falta de comunicação (Siddique \& Hussein, 2019).

\subsubsection{Coleta e entendimento dos requisitos dos clientes}

A coleta e análise de requisitos dos clientes é atividade crítica no desenvolvimento de software e quando se fala em filosofia ágil isso normalmente envolve as partes interessadas na escrita destes requisitos em formato de histórias de usuário (Maiden \& Jones, 2010), exigindo uma colaboração muito próxima entre o cliente e o time de desenvolvimento. Porém, 
devido à complexidade desta atividade, existem desafios significativos na obtenção de requisitos de qualidade (Cao, Mohan, Xu, \& Ramesh, 2009; Ovaska, Rossi, \& Smolander, 2005) e por isso essa é uma variável importante quando se fala em foco no cliente. Neste contexto, foi constituída a seguinte hipótese:

- Hipótese H1. A coleta e entendimento de requisitos (CER) influencia positivamente o foco no cliente (FC) em times ágeis de desenvolvimento.

\subsubsection{Coleta e utilização de informação do cliente}

De acordo com Coltman (2007), organizações de sucesso coletam proativamente dados de seus clientes a partir de necessidades articuladas ou latentes, o que difere da coleta e entendimento dos requisitos dos clientes, pois são dados não manifestados explicitamente durante essa fase. Portanto, obter o máximo de dados possível sobre o cliente ajuda o time de desenvolvimento a entender os problemas e oportunidades existentes, desenvolvendo sistemas de qualidade do ponto de vista do próprio cliente (Zultner, 1993). Com base neste contexto, foi estabelecida a seguinte hipótese:
- Hipótese H2. A coleta e utilização de informação do cliente (CUID) influencia positivamente o foco no cliente (FC) em times ágeis de desenvolvimento.

\subsubsection{Recebendo e utilizando feedback do cliente}

O feedback do cliente deve ser utilizado para treinar os times de desenvolvimento e melhorar processos onde é necessário e isso é chamado de capacidade de desenvolvimento (Gulati, 2007), o que é essencial para que as pessoas tenham as competências necessárias para entregar soluções focadas no cliente e melhorar produtos e processos em geral (Bragge \& Merisalo-Rantanen, 2009).

Nos métodos ágeis a obtenção de feedback do cliente é facilitada por meio das reuniões de revisão e retrospectiva de maneira constante, o que também é atividade crítica no processo de desenvolvimento em times ágeis (Chamberlain, Sharp, \& Maiden, 2006; Lindvall, Muthig, Dagnino, Wallin, Stupperich, Kiefer, May, \& Kahkonen, 2004). Deste modo, as informações supracitadas dão suporte para a seguinte hipótese: 
- Hipótese H3. O recebimento e uso de feedback do cliente (RUFC) influencia positivamente o foco no cliente (FC) em times ágeis de desenvolvimento.

\subsubsection{Melhorando o relacionamento com o cliente}

O relacionamento com o cliente difere do processo de feedback porque envolve negociações e sentimentos entre as pessoas. Melhorar o relacionamento com o cliente deveria ser parte do processo de desenvolvimento, influenciando a maneira como os sistemas são concebidos (Liang \& Tanniru, 2006; Parzinger \& Nath, 2000; Sousa, 2003).

Além de bom relacionamento, a disponibilidade e competência dos participantes de projetos de desenvolvimento de software por parte do cliente são fatores essenciais para garantir o sucesso ( Grimstad, Jørgensen, \& Moløkken-Østvold, 2006). Com base nestas informações a seguinte hipótese foi elaborada:

- Hipótese H4. A melhoria no relacionamento com o cliente (MRC) influencia positivamente o foco no cliente (FC) em times ágeis de desenvolvimento.

\subsubsection{Foco no cliente}

Empresas com orientação para o cliente são mais inovadoras e conseguem desenvolver vantagem competitiva, com melhor desempenho no geral falando de indicadores financeiros e não financeiros (Grinstein, 2008; Hult \& Ketchen Jr, 2001; Kirca et al., 2011; Kirca et al., 2005). A Figura 1 demonstra as relações construídas a partir da revisão de literatura. 
Figura 1.

Modelo de Pesquisa e Hipóteses

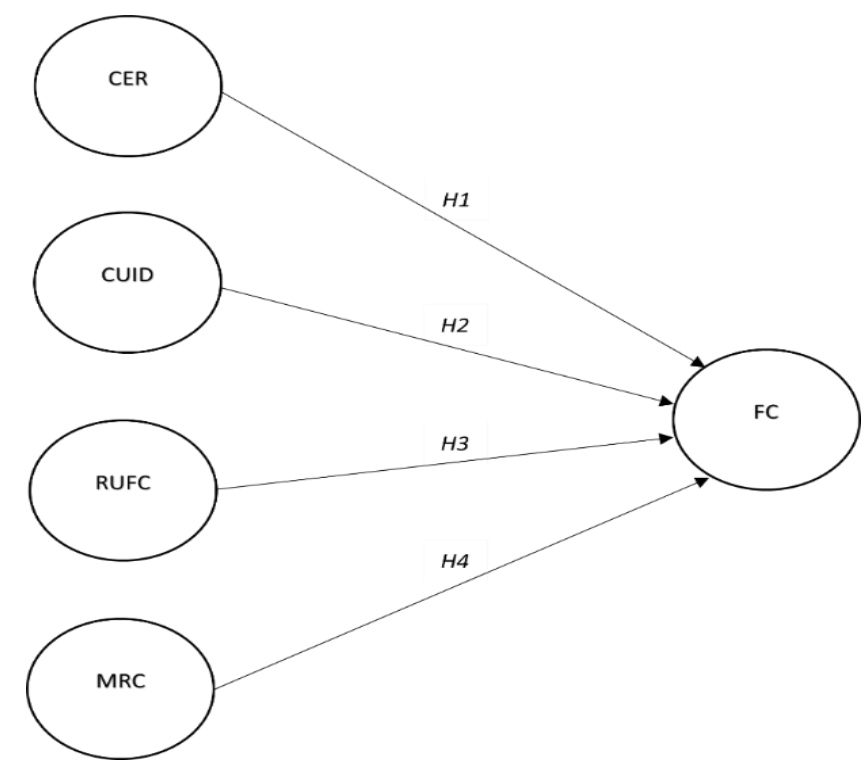

Fonte: Elaborada pelos autores, 2021.

Portanto, o conteúdo apresentado permite a melhor compreensão da orientação para o cliente, que pode ser dividida em responsiva e proativa, onde a primeira é baseada nas informações disponíveis sobre o cliente e a segunda na descoberta e atendimento de necessidades latentes não expressadas, ajudando assim a criar produtos e serviços que satisfaçam os clientes (Hair Jr et al., 2014).

\section{Método de pesquisa}

Esta pesquisa avaliou se o estudo sobre foco no cliente de Lohan et al. (2011) é estatisticamente significativo e em que medida cada um dos construtos do modelo criado pelos autores influencia o foco no cliente, no Brasil. O método escolhido para validação do modelo é o PLS-PM, indicado para confirmação de teorias com base em modelos hierárquicos (Chin, 1998), que exige amostra completa com distribuição normal para validação do modelo (Faul, Erdfelder, Lang, \& Buchner, 2007).

\subsection{Método de coleta de dados}

Esta pesquisa utilizou amostra de conveniência porque o objetivo foi obter respostas de indivíduos que trabalham ou trabalharam com métodos ágeis de desenvolvimento no Brasil, o que vai de encontro à experiência prévia do autor e por isso utilizou-se como principal estratégia a divulgação da pesquisa através de rede 
social de trabalho, facilitando assim a seleção geral da amostra ( Etikan, Musa, \& Alkassim, 2016).

A pesquisa foi realizada no nível de indivíduos, que possuem experiência com métodos ágeis e atuam nas funções de: CEOs, diretores, gerentes, gerentes de projeto, product owners, scrum masters e membros de times de desenvolvimento. A pesquisa ficou vigente de novembro de 2020 a fevereiro de 2021, o número total de respondentes foi de 152 pessoas sendo que 91 questionários foram respondidos de maneira completa e puderam ser considerados válidos.

\subsection{Questionário}

Após a revisão de literatura, com base nas conclusões do estudo de Lohan et al. (2011) foi elaborado o instrumento de pesquisa, onde foram avaliadas 23 questões relacionadas às variáveis dependentes: coleta e entendimento de requisitos (CER), coleta e uso de informações do cliente (CUID), recebimento e utilização de feedback do cliente (RUFC), melhoria no relacionamento com o cliente (MRC) e foco no cliente (FC), como apresentado na Tabela 2.

Para validação do instrumento de pesquisa foi realizado pré-teste com 12 alunos do mestrado profissional em gestão para competitividade na linha de TI, durante o mês anterior à disponibilização do questionário oficial. Os respondentes do pré-teste trabalham com a gestão de times ágeis de desenvolvimento, conhecem este campo de pesquisa e foram capazes de analisar e sugerir melhorias na clareza, conteúdo e tempo de resposta de cada questão. Além disso, foram analisadas estatisticamente a validade e confiabilidade do instrumento de pesquisa (Hair et al., 2011; Moore \& Benbasat, 1991), conforme são apresentados nas Tabela 3 e Tabela 4.

\section{Tabela 2.}

Questões Referentes às Variáveis Independentes

\begin{tabular}{|c|l|l|}
\hline Variável & \multicolumn{1}{|c|}{ Questão } & \multicolumn{1}{|c|}{ Referência } \\
\hline RUFC_1 & $\begin{array}{l}\text { O time de desenvolvimento sempre recebe feedback sobre a } \\
\text { satisfação do cliente }\end{array}$ & $\begin{array}{l}\text { (Moe, Dingsøyr, \& } \\
\text { Dybå, 2010) }\end{array}$ \\
\hline RUFC_2 & $\begin{array}{l}\text { O feedback do cliente é utilizado para treinar os membros do time de } \\
\text { desenvolvimento }\end{array}$ & (Moe et al., 2010) \\
\hline RUFC_3 & $\begin{array}{l}\text { As reuniões de retrospectiva sempre geram oportunidades de } \\
\text { melhoria }\end{array}$ & $\begin{array}{l}\text { (Lohan } \text { et al., } \\
\text { 2011) }\end{array}$ \\
\hline RUFC_4 & O cliente sempre comparece às reuniões de retrospectiva do time & $\begin{array}{l}\text { (Lohan } \text { et al., } \\
\text { 2011) }\end{array}$ \\
\hline
\end{tabular}


Ribeiro, F. C., Larieira, C. L. C., Yoshikuni, A. C., \& Costa, I. (2021, set./dez.). Foco no cliente em times de desenvolvimento de software: um estudo quantitativo

\begin{tabular}{|c|c|c|}
\hline Variável & Questão & Referência \\
\hline MRC_1 & $\begin{array}{l}\text { O cliente está diretamente envolvido no processo de } \\
\text { desenvolvimento, acompanhando a progressão do projeto }\end{array}$ & (Kautz, 2009) \\
\hline MRC_2 & O time de desenvolvimento tem reuniões frequentes de sprint review & $\begin{array}{l}\text { (Lohan et al., } \\
\text { 2011) }\end{array}$ \\
\hline MRC_3 & O cliente participa de todas as reuniões de sprint review & $\begin{array}{l}\text { (Lohan et al., } \\
\text { 2011) }\end{array}$ \\
\hline CUID_1 & $\begin{array}{l}\text { O time de desenvolvimento recebe com frequência treinamento sobre } \\
\text { o negócio da empresa }\end{array}$ & $\begin{array}{l}\text { (Lohan et al., } \\
\text { 2011) }\end{array}$ \\
\hline CUID_2 & Existem mecanismos para disseminar o conhecimento sobre o cliente & (Coltman, 2007) \\
\hline CUID_3 & $\begin{array}{l}\text { Nós coletamos e armazenamos dados de nossos clientes para ajudar } \\
\text { em futuras tomadas de decisão }\end{array}$ & (Coltman, 2007) \\
\hline CUID_4 & $\begin{array}{l}\text { Informações sobre o cliente são coletadas e analisadas antes mesmo } \\
\text { dos projetos iniciarem }\end{array}$ & $\begin{array}{l}\text { (Lohan et al., } \\
\text { 2011) }\end{array}$ \\
\hline CUID_5 & $\begin{array}{l}\text { O time de desenvolvimento é incentivado a compartilhar informações } \\
\text { sobre o cliente }\end{array}$ & (Coltman, 2007) \\
\hline CER_1 & As histórias de usuário guiam todo o processo de desenvolvimento & $\begin{array}{l}\text { (Lohan et al., } \\
\text { 2011) }\end{array}$ \\
\hline CER_2 & $\begin{array}{l}\text { As histórias de usuário sempre estão disponíveis durante o processo } \\
\text { de desenvolvimento, quando necessário }\end{array}$ & $\begin{array}{l}\text { (Lohan et al., } \\
\text { 2011) }\end{array}$ \\
\hline CER_3 & $\begin{array}{l}\text { O time de desenvolvimento recebe histórias de usuário suficientes } \\
\text { para entregar o que o cliente precisa }\end{array}$ & $\begin{array}{l}\text { (Lohan et al., } \\
\text { 2011) }\end{array}$ \\
\hline CER_4 & $\begin{array}{l}\text { A relação entre o cliente e o time de desenvolvimento ajuda na } \\
\text { qualidade das entregas }\end{array}$ & $\begin{array}{l}\text { (Lohan et al., } \\
\text { 2011) }\end{array}$ \\
\hline CER_5 & $\begin{array}{l}\text { A relação entre o cliente e o time de desenvolvimento ajuda no prazo } \\
\text { de entrega }\end{array}$ & $\begin{array}{l}\text { (Lohan et al., } \\
\text { 2011) }\end{array}$ \\
\hline FC_1 & $\begin{array}{l}\text { Incentivamos os funcionários a desenvolver novos conceitos de } \\
\text { produtos }\end{array}$ & $\begin{array}{l}\text { (Hair Jr et al., } \\
\text { 2014) }\end{array}$ \\
\hline FC_2 & $\begin{array}{l}\text { Nós garantimos que o nosso negócio existe principalmente para servir } \\
\text { os clientes }\end{array}$ & $\begin{array}{l}\text { (Hair Jr et al., } \\
\text { 2014) }\end{array}$ \\
\hline FC_3 & $\begin{array}{l}\text { Trabalhamos para sermos mais focados nos clientes do que nossos } \\
\text { concorrentes }\end{array}$ & $\begin{array}{l}\text { (Hair Jr et al., } \\
\text { 2014) }\end{array}$ \\
\hline FC_4 & $\begin{array}{l}\text { Nós monitoramos constantemente o nível de compromisso para servir } \\
\text { as necessidades dos nossos clientes }\end{array}$ & $\begin{array}{l}\text { (Hair Jr et al., } \\
\text { 2014) }\end{array}$ \\
\hline FC_5 & Nós muitas vezes testamos novos produtos em mercados selecionados & $\begin{array}{l}\text { (Hair Jr et al., } \\
\text { 2014) }\end{array}$ \\
\hline FC_6 & $\begin{array}{l}\text { Procuramos oportunidades em áreas onde os clientes têm dificuldade } \\
\text { em expressar suas necessidades }\end{array}$ & $\begin{array}{l}\text { (Hair Jr et al., } \\
\text { 2014) }\end{array}$ \\
\hline
\end{tabular}

Fonte: Elaborado pelos autores, 2021.

\subsection{Definição de escala e validação do questionário}

Todas as questões foram elaboradas utilizando escala Likert de 5 pontos, indo de "discordo totalmente" à "concordo totalmente". A escolha por uma escala de 5 pontos favorece a facilidade nas respostas e mantém um ponto neutro evitando a falta de distinção clara entre os pontos 2 e 3 , na ausência deste item (Júnior \& Costa, 2014).

As questões não possuem escala negativa e estão relacionadas cada uma à uma única ideia evitando o uso de condicionais, fazendo uso de palavras simples e termos claros para seu públicoalvo e são descritas de maneira afirmativa, evitando dúvidas nos respondentes 
(Nemoto \& Beglar, 2014). Cada construto deve possuir validade convergente e discriminante de maneira a garantir que cada item do questionário está associado de maneira correta ao seu construto e que a escala é válida (Moore \& Benbasat, 1991).

\subsection{Características da amostra}

Para calcular o tamanho mínimo da amostra foi utilizado o software $\mathrm{G}^{*}$ Power, tendo como parâmetros para o cálculo: effect size $\left(\mathrm{f}^{2}\right)=0.15$, err prob $(\alpha)=0.05$, power $(1-\beta)=0.80$ e number of tested predictors $=5$. Como resultado foi calculada a amostra mínima de 85 respostas válidas com poder estatístico de $80 \%$ (Faul et al., 2007). Após a disponibilização do questionário eletrônico foram obtidas 91 repostas completas, o que representa um poder estatístico de $83,4 \%$.

\subsection{Modelo de medição}

O método utilizado para validação do modelo foi o PLS-PM, recomendado para testar e validar modelos exploratórios, evitando problemas possíveis com amostras menores (Wold, 1974, 1982). A validação de resultados com PLS-PM é feita através dos modelos interno e externo, onde o modelo interno é representado pela relação entre as variáveis latentes e o externo pela relação entre as variáveis exógenas e suas variáveis latentes (Chin, 1998).

Foram executados testes de validação do modelo através da técnica de bootstrapping, que consiste na geração de amostras aleatórias a partir da amostra original, reestimando o modelo para cada uma destas amostras na tentativa de garantir a consistência dos resultados obtidos (Hair et al., 2009). Para este modelo foram geradas 1000 amostras aleatórias e o desvio padrão é apresentado no modelo estrutural.

Para a validade discriminante foi utilizado como critério a carga fatorial cruzada entre todos os construtos e suas variáveis observadas, garantindo que nenhuma variável tem carga fatorial maior em um construto que não ao qual está associado, como apresentado na Tabela 3 (Fornell \& Larcker, 1981). 
Tabela 3.

Carga Fatorial Cruzada

\begin{tabular}{lcccccc}
\hline Variável & Itens & CER & CUIC & RUFC & MRC & FC \\
\hline \multirow{4}{*}{ Coleta e entendimento de requisitos (CER) } & CER_1 & $\mathbf{0 , 8 8 4 2 0 0}$ & 0,350822 & 0,294238 & 0,247684 & 0,483817 \\
& CER_2 & $\mathbf{0 , 7 2 2 7 1 2}$ & 0,126981 & 0,258888 & 0,228780 & 0,269976 \\
& CER_3 & $\mathbf{0 , 7 9 4 9 3 7}$ & 0,210902 & 0,257154 & 0,010659 & 0,337233 \\
& CER_4 & $\mathbf{0 , 6 4 9 9 9 0}$ & 0,330536 & 0,600485 & 0,485454 & 0,501043 \\
\hline \multirow{4}{*}{$\begin{array}{l}\text { Coleta e utilização de informações do } \\
\text { cliente (CUIC) }\end{array}$} & CUIC_1 & 0,117526 & $\mathbf{0 , 5 9 4 0 6 3}$ & 0,320162 & 0,086791 & 0,394347 \\
& CUIC_2 & 0,361642 & $\mathbf{0 , 8 2 8 9 8 3}$ & 0,455263 & 0,039530 & 0,657685 \\
& CUI__3 & 0,284394 & $\mathbf{0 , 7 9 0 0 0 4}$ & 0,310533 & $-0,053896$ & 0,487486 \\
& CUIC_4 & 0,264023 & $\mathbf{0 , 7 3 3 8 6 2}$ & 0,296714 & 0,113145 & 0,514341 \\
\hline \multirow{3}{*}{$\begin{array}{l}\text { Recebendo e utilizando feedback do } \\
\text { cliente (RUFC) }\end{array}$} & RUFC_1 & 0,338033 & 0,346805 & $\mathbf{0 , 7 1 4 6 7 6}$ & 0,382518 & 0,339019 \\
& RUFC_2 & 0,163931 & 0,320676 & $\mathbf{0 , 7 0 1 3 7 9}$ & 0,248232 & 0,396908 \\
& RUFC_3 & 0,511820 & 0,329441 & $\mathbf{0 , 6 8 9 0 5 3}$ & 0,278304 & 0,447634 \\
\hline \multirow{2}{*}{$\begin{array}{l}\text { Melhorando o relacionamento com o } \\
\text { cliente (MRC) }\end{array}$} & MRC_1 & 0,339559 & 0,135786 & 0,347216 & $\mathbf{0 , 8 7 6 9 9 8}$ & 0,258432 \\
& MRC_2 & 0,105806 & $-0,087689$ & 0,292103 & $\mathbf{0 , 5 5 3 7 7 1}$ & 0,127503 \\
& MRC_3 & 0,267976 & $-0,055048$ & 0,254507 & $\mathbf{0 , 6 1 5 5 6 4}$ & 0,066955 \\
\hline \multirow{2}{*}{ Foco no cliente (FC) } & FC_1 & 0,383487 & 0,504459 & 0,505284 & 0,213000 & $\mathbf{0 , 7 4 6 7 9 0}$ \\
& FC_2 & 0,403896 & 0,418285 & 0,372177 & 0,232140 & $\mathbf{0 , 7 2 6 0 2 5}$ \\
& FC_3 & 0,454293 & 0,561517 & 0,421886 & 0,249373 & $\mathbf{0 , 7 7 0 9 7 2}$ \\
& FC_4 & 0,440038 & 0,609736 & 0,464852 & 0,232053 & $\mathbf{0 , 8 1 1 6 7 3}$ \\
& FC_5 & 0,253761 & 0,433335 & 0,205068 & $-0,043383$ & $\mathbf{0 , 5 5 3 6 6 1}$ \\
& FC_6 & 0,431730 & 0,518848 & 0,455226 & 0,177088 & $\mathbf{0 , 7 0 9 0 9 6}$ \\
\hline
\end{tabular}

Fonte: Elaborada pelos autores, 2021.

Como critério para análise da validade convergente foi utilizado o AVE (average variance extracted), que deve ser no mínimo 0,50 para cada construto, isto é, a variável latente deve explicar em média mais da metade da variância de seus indicadores (Fornell \& Larcker, 1981). Para medir o grau de consistência entre os indicadores dos construtos foi utilizado como critério o $\mathrm{CR}$ (composite reliability), indicador recomentado para pesquisas que utilizam o método PLS-PM (Werts, Linn, \& Jöreskog, 1974) e que assim como o Alpha de Cronbach, indicador amplamente utilizado em métodos quantitativos, deve apresentar um valor acima de 0,70 (Fronell, 1982; Tenenhaus, Vinzi, Chatelin, \& Lauro, 2005).

A Tabela 4 apresenta os valores de AVE e CR para todos os construtos, além da raiz quadrada da AVE de cada construto na diagonal, garantindo que não há relação 
maior da variável latente com outra variável

latente (Fornell \& Larcker, 1981).

\section{Tabela 4.}

Validação das Variáveis Latentes

\begin{tabular}{lccccc}
\hline Construtos & $\mathbf{1}$ & $\mathbf{2}$ & $\mathbf{3}$ & $\mathbf{4}$ & $\mathbf{5}$ \\
\hline 1. CER & $\mathbf{0 , 7 6 7 8 7 6}$ & & & & \\
2. CUID & 0,362264 & $\mathbf{0 , 7 4 2 0 8 9}$ & & & \\
3. RUFC & 0,493023 & 0,473180 & $\mathbf{0 , 7 0 1 7 8 1}$ & & \\
4. MRC & 0,352134 & 0,059755 & 0,425495 & $\mathbf{0 , 6 9 6 3 4 9}$ & \\
5. FC & 0,552116 & 0,707014 & 0,570302 & 0,258599 & $\mathbf{0 , 7 2 4 2 6 7}$ \\
Composite reliability $(C R)$ & 0,850165 & 0,828534 & 0,744287 & 0,730446 & 0,867318 \\
Average variance extracted $(A V E)$ & 0,589633 & 0,550696 & 0,492496 & 0,484902 & 0,524563 \\
\hline
\end{tabular}

Fonte: Elaborada pelos autores, 2021.

\subsection{Modelo estrutural}

A análise do modelo estrutural em PLS-PM é apresentada na Tabela 5, contendo o tamanho do efeito $\left(\mathrm{f}^{2}\right)$, coeficiente de caminho, $\mathrm{R}^{2}$ ajustado, valor $p$, valor $t$, desvio padrão em relação ao bootstrap e valor de inflação da variância (VIF).

Com base nos resultados, evidenciou que duas variáveis latentes apresentaram valor de AVE abaixo do recomendado $(0,50)$, porém como o valor para o construto RUFC ficou muito próximo do recomendado e o $\mathrm{CR}$ apresentou valor adequado a variável foi mantida no modelo, confirmando-se sua relação com o FC.

De acordo com Hair et al. (2011) deve ser considerada a retirada de variáveis independentes com carga fatorial abaixo de 0,70 desde que sua retirada contribua para a melhora da confiabilidade do modelo, o que foi o caso para 3 variáveis: CER_5, CUID_5 e RUFC_4, que apresentaram as seguintes cargas fatoriais, respectivamente: 0,66, 0,62 e 0,53. Por esta razão estas variáveis são apresentadas na Tabela 2 , mas não estão presentes na Tabela 3. A retirada destas variáveis do modelo melhorou o $\mathrm{R}^{2}$ de 0,599 para 0,611 . 


\section{Tabela 5.}

Análise de Significância e Efeitos

\begin{tabular}{lcccccccccc}
\hline $\begin{array}{l}\text { Relacionamento } \\
\text { entre variáveis }\end{array}$ & Hipótese & $\begin{array}{c}\text { Hipótese } \\
\text { suportad } \\
\mathbf{a}\end{array}$ & VIF & $\mathbf{f}^{2}$ & $\begin{array}{c}\text { Coeficiente } \\
\mathbf{d e} \\
\mathbf{c a m i n h o} \\
(\boldsymbol{\beta})\end{array}$ & $\begin{array}{c}\text { Desvio } \\
\text { padrão }\end{array}$ & valor t & $\begin{array}{c}\mathbf{R}^{2} \\
\text { valor p } \\
\text { ajust } \\
\text { ado }\end{array}$ \\
\hline CER -> FC & H1 (+) & Sim & 1,427 & 0,1198 & 0,2544 & 0,0833 & 3,0523 & 0,0010 & \\
CUID -> FC & H2 (+) & Sim & 1,390 & 0,5394 & 0,5333 & 0,0700 & 7,6180 & 0,0000 & 0,611 \\
RUFC -> FC & H3 (+) & Sim & 1,754 & 0,0405 & 0,1638 & 0,0723 & 2,2649 & 0,0130 & \\
MRC -> FC & H4 (+) & Não & 1,323 & 0,0091 & 0,0674 & 0,0799 & 0,8442 & 0,2000 & \\
\hline
\end{tabular}

Fonte: Elaborada pelos autores

A avaliação do modelo estrutural é feita através do $\mathrm{R}^{2}$, coeficiente de determinação das variáveis latentes endógenas, que no caso da variável FC atingiu o índice de 0,628 , sendo o valor ajustado igual a 0,611. De acordo com Hair et al. (2011) os valores de 0,75, 0,50 e 0,25 significam efeitos substancial, moderado e fraco, respectivamente, outros autores consideram que estes valores seriam 0,67 , 0,33 e 0,19 (Chin, 1998), portanto podemos considerar o resultado de $\mathrm{R}^{2}$ sobre a variável FC como de moderado a substancial.
Outra medida de adequação de modelo amplamente utilizada em PLS-PM é o GoF (goodness-of-fit), que para este modelo apresentou um resultado de 0,55. Segundo Wetzels, Odekerken-Schröder e Van Oppen (2009) os valores de 0,36, 0,25 e 0,1 podem ser considerados alto, médio e fraco, respectivamente, portanto podemos considerar o modelo de maneira geral adequado. $\mathrm{O}$ modelo geral e hipóteses são ilustrados na Figura 2. 


\section{Figura 2.}

Modelo de Pesquisa com Coeficientes de Caminho ( $\beta)$ e Cargas Fatoriais

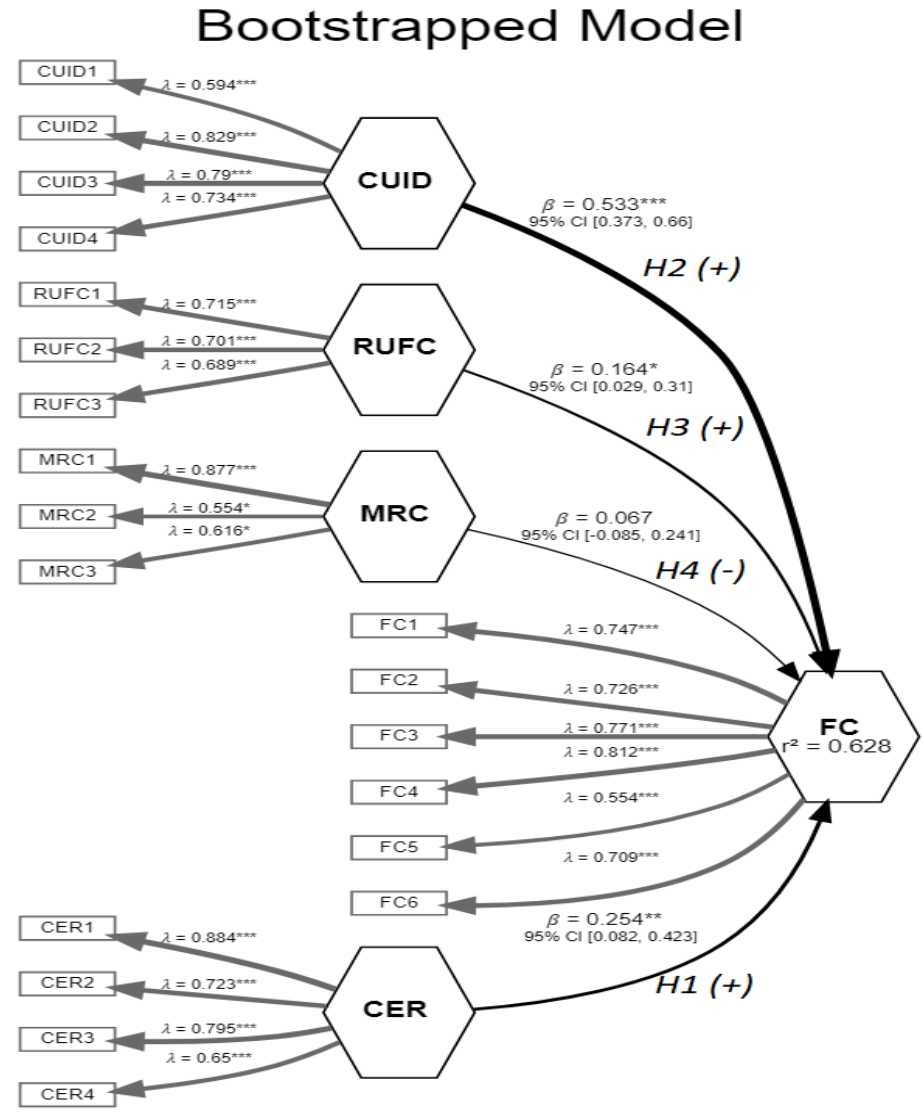

Fonte: Elaborada pelos autores, 2021.

\section{Discussão dos resultados}

Como o estudo de Lohan et al. (2011) sobre foco no cliente foi baseado em estudo de caso múltiplo realizado na Irlanda e Noruega e não foram encontrados outros estudos para validação no Brasil, a contribuição desta pesquisa é neste sentido. Além de validar o modelo, o resultado obtido pode indicar para profissionais que implementam ou que estão tendo dificuldades com métodos ágeis em suas organizações, quais são os fatores mais relevantes para que consigam ter foco no cliente, atingindo um dos principais objetivos destes métodos, que é a satisfação do cliente (Gandomani \& Nafchi, 2016).

O resultado da relação entre as variáveis coleta e entendimento de requisitos (CER), coleta e utilização de informações do cliente (CUID), recebendo e utilizando feedback do cliente (RUFC), melhorando o relacionamento com o cliente 
(MRC) e o foco no cliente (FC) é apresentado na seção 3.6, por meio do coeficiente de caminho e tamanho do efeito para cada uma delas, avaliando as hipóteses apresentadas na seção 2.5 .

A hipótese $\mathrm{H} 1$ foi suportada $(\beta=$ $0,254$, valor $\mathrm{t}=3,0523$ e valor $\mathrm{p}=0,001) \mathrm{e}$ os resultados demonstram que a coleta e entendimento de requisitos (CER) influencia positivamente o foco no cliente (FC) em times ágeis de desenvolvimento e as questões com maior efeito de mensuração, capazes de identificar as principais práticas, são:

- CER_1. As histórias de usuário guiam todo $\mathrm{o}$ processo $\mathrm{de}$ desenvolvimento $(\beta=0,884)$;

- CER_3. O time de desenvolvimento recebe histórias de usuário suficientes para entregar o que o cliente precisa $(\beta$ $=0,795)$;

- CER_2. As histórias de usuário sempre estão disponíveis durante o processo de desenvolvimento, quando necessário $(\beta=0,723)$.

A hipótese $\mathrm{H} 2$ foi fortemente suportada $(\beta=0,533$, valor $\mathrm{t}=7,618$ e valor $\mathrm{p}=0,000)$ e os resultados demonstram que a coleta e utilização de informação do cliente (CUID) é o fator com maior efeito de mensuração no foco no cliente (FC) em times ágeis de desenvolvimento e as questões com maior efeito de mensuração, capazes de identificar as principais práticas, são:

- CUID_2. Existem mecanismos para disseminar o conhecimento sobre o cliente $(\beta=0,829)$;

- CUID_3. Nós coletamos e armazenamos dados de nossos clientes para ajudar em futuras tomadas de decisão $(\beta=0,79)$;

- CUID_4. Informações sobre o cliente são coletadas e analisadas antes mesmo dos projetos iniciarem $(\beta=0,734)$.

A hipótese H3 apresentou AVE pouco abaixo de 0,5 , porém com $\mathrm{CR}$ elevado de 0,744 , coeficiente de caminho, valor $\mathrm{t}$ e valor $\mathrm{p}$ relevantes $(\beta=0,164$, valor $t=2,265$ e valor $p=0,013)$, por isso foi considerada suportada indicando que o recebimento e uso de feedback do cliente (RUFC) influencia positivamente o foco no cliente (FC) em times ágeis de desenvolvimento e as questões com maior efeito de mensuração, capazes de identificar as principais práticas, são:

- RUFC_1. O time de desenvolvimento sempre recebe 
feedback sobre a satisfação do cliente $(\beta=0,715)$;

- RUFC_2. O feedback do cliente é utilizado para treinar os membros do time de desenvolvimento $(\beta=$ $0,701)$.

A hipótese $\mathrm{H} 4$ não foi suportada $(\beta=$ 0,067 , valor $\mathrm{t}=0,844$ e valor $\mathrm{p}=0,2$ ), pois apresenta valor $\mathrm{p}>0,05$ e AVE $<0,5$, identificando que a variável não é capaz de explicar mais da metade da variância de seus indicadores, além disso o próprio coeficiente de caminho apresenta valor muito abaixo das outras variáveis, portanto considera-se que são necessários estudos futuros sobre a melhoria no relacionamento com o cliente (MRC) e sua influência no foco no cliente (FC) em times ágeis de desenvolvimento.

\section{Conclusões}

O objetivo deste estudo foi atingido ao avaliar os efeitos das variáveis coleta e entendimento de requisitos, coleta e utilização de informações do cliente, recebendo e utilizando feedback do cliente e melhorando o relacionamento com o cliente no foco no cliente em times ágeis de desenvolvimento, no Brasil, dado que um dos princípios do manifesto ágil é satisfazer o cliente.

$O$ resultado demonstra que nem todos os fatores analisados têm relevância no foco no cliente e que melhorar o relacionamento com o cliente pode ser importante em outros aspectos, mas a hipótese de que isso influencia o foco no cliente em times ágeis de desenvolvimento não foi comprovada nesta pesquisa.

Foram avaliados quatro fatores que influenciam o foco no cliente em times ágeis de desenvolvimento: coleta e entendimento de requisitos, coleta $\mathrm{e}$ utilização de informações do cliente, recebimento e utilização de feedback do cliente e melhoria no relacionamento com o cliente. O resultado indica para os profissionais que implementam métodos ágeis em suas organizações quais os fatores com maior relevância e onde eles deveriam concentrar seus esforços para ter foco no cliente e satisfazê-lo.

Ao focar na coleta e utilização de informações do cliente, são boas práticas coletar estes dados antes e durante os projetos, armazenando-os para futuras tomadas de decisão e disseminando o conhecimento sobre o cliente para os times de desenvolvimento ágil. Outro fator relevante é a coleta e entendimento de requisitos dos clientes, que é parte crítica no processo de desenvolvimento, independentemente do método implementado, mas que no caso de métodos ágeis deve ser feita através de escrita em 
conjunto com o cliente na forma de histórias de usuário, estas histórias de usuário devem guiar todo o processo de desenvolvimento, devem ser suficientes para entregar o que o cliente precisa e precisam estar disponíveis durante todo $\mathrm{o}$ processo de desenvolvimento. Outra indicação deste estudo é que as organizações deveriam se preocupar em receber e utilizar de maneira adequada o feedback do cliente, para treinar os membros dos times de desenvolvimento, solicitando com frequência este feedback.

Este estudo apresenta limitações e uma delas é a dificuldade de calcular os efeitos moderadores mencionados por Lohan et al. (2011) em seu estudo de caso, devido ao tamanho da amostra e do questionário, onde 91 respostas foram completas, dos 152 respondentes no total. Assim, como recomendação para estudos futuros seria interessante avaliar os efeitos moderadores identidade do cliente, localização do cliente, personalidade percebida do cliente e experiência anterior do time de desenvolvimento propostos por Lohan et al. (2011) em cada um dos construtos apresentados nesta pesquisa, através de questionário específico e com foco nos efeitos moderadores.

Outra recomendação de estudo futuro seria no sentido de entender quais fatores realmente influenciam a melhoria no relacionamento com o cliente, para então verificar se este tem influência ou não de maneira significativa no foco no cliente em times ágeis de desenvolvimento. Por último, seria recomendado avaliar de maneira qualitativa como as empresas que têm foco no cliente no processo de desenvolvimento ágil tem feito a coleta e uso de informações do cliente para obter sucesso.

\section{Referências}

Ayabakan, S., Bardhan, I. R., \& Zheng, Z. (2017). A data envelopment analysis approach to estimate itenabled production capability. MIS Quarterly, 41(1), A1-A7.

Beck, K. (1999). Embracing change with extreme programming. Computer, 32(10), 70-77.

Beck, K., Cunningham, W., Hunt, A., Martin, R., Thomas, D., Beedle, M., \& Sutherland, J. (2001). Manifesto ágil. Manifesto para Desenvolvimento Ágil de Software.

Boehm, B. (2002). Get ready for agile methods, with care. Computer, 35(1), 64-69.

Bragge, J., \& Merisalo-Rantanen, H. (2009). Engineering e-collaboration processes to obtain innovative enduser feedback on advanced webbased information systems. Journal of the Association for Information Systems, 10(3), 196-220.

Budacu, E. N., \& Pocatilu, P. (2018). Real Time Agile Metrics for Measuring 
Team Performance. Informatica Economica, 22(4), 70-79.

Cao, L., Mohan, K., Xu, P., \& Ramesh, B. (2009). A framework for adapting agile development methodologies. European Journal of Information Systems, 18(4), 332-343.

Chamberlain, S., Sharp, H., \& Maiden, N. (2006). Towards a framework for integrating agile development and user-centred design. International Conference on Extreme Programming and Agile Processes in Software Engineering, Oulu, Finlâdia.

Chin, W. W. (1998). The partial least squares approach to structural equation modeling. Modern Methods for Business Research, 295(2), 295-336.

Cohen, D., Lindvall, M., \& Costa, P. (2004). An introduction to agile methods. Adv. Comput., 62(03), 166.

Collyer, S., Warren, C., Hemsley, B., \& Stevens, C. (2010). Aim, fire, aim-Project planning styles in dynamic environments. Project Management Journal, 41(4), 108121.

Coltman, T. (2007). Why build a customer relationship management capability? The Journal of Strategic Information Systems, 16(3), 301320.

Denning, S. (2019). Lessons learned from mapping successful and unsuccessful Agile transformation journeys. Strategy \& Leadership, 47(44).
Etikan, I., Musa, S. A., \& Alkassim, R. S. (2016). Comparison of convenience sampling and purposive sampling. American Journal of Theoretical and Applied Statistics, 5(1), 1-4.

Faul, F., Erdfelder, E., Lang, A.-G., \& Buchner, A. (2007). G* Power 3: A flexible statistical power analysis program for the social, behavioral, and biomedical sciences. Behavior Research Methods, 39(2), 175-191.

Fornell, C., \& Larcker, D. F. (1981). Evaluating structural equation models with unobservable variables and measurement error. Journal of Marketing Research, 18(1), 39-50.

Fronell, C. (1982). A second generation of multivariate analysis methods. New York: Praeger. and Fred L. Bookstein (1982)," Two Structural Equation Models: LISREL and PLS Applied to Consumer Exit-Voice Theory," Journal of Marketing Research, 19, 440-452.

Gandomani, T. J., \& Nafchi, M. Z. (2016). The essential prerequisites of Agile transition and adoption: A grounded theory approach. Journal of Internet Computing and Services, 17(5), 173-184.

Ghani, I., Bello, M., \& Bagiwa, I. L. (2015). A Survey-based Analysis of Agile Adoption on Performances of IT Organizations. Journal of Internet Computing and Services, 16(5), 87-92.

Grimstad, S., Jørgensen, M., \& MoløkkenØstvold, K. (2006). Software effort estimation terminology: The tower of Babel. Information and Software Technology, 48(4), 302-310. 
Grinstein, A. (2008). The effect of market orientation and its components on innovation consequences: a metaanalysis. Journal of the Academy of Marketing Science, 36(2), 166-173.

Gulati, R. (2007). Silo busting. Harvard Business Review, 85(5), 98-108.

Hair, J. F., Black, W. C., Babin, B. J., Anderson, R. E., \& Tatham, R. L. (2009). Análise multivariada de dados. Bookman editora.

Hair, J. F., Ringle, C. M., \& Sarstedt, M. (2011). PLS-SEM: Indeed a silver bullet. Journal of Marketing Theory and Practice, 19(2), 139-152.

Hair Jr, J. F., Gabriel, M. L., \& Patel, V. K. (2014). Modelagem de Equações Estruturais Baseada em Covariância (CB-SEM) com o AMOS: Orientações sobre a sua aplicação como uma Ferramenta de Pesquisa de Marketing. Revista Brasileira de Marketing, 13(2), 4455.

Hult, G. T. M., \& Ketchen Jr, D. J. (2001). Does market orientation matter? A test of the relationship between positional advantage and performance. Strategic Management Journal, 22(9), 899906.

Júnior, S. D. d. S., \& Costa, F. J. (2014). Mensuração e escalas de verificação: uma análise comparativa das escalas de Likert $\mathrm{e}$ Phrase Completion. PMKT-Revista Brasileira de Pesquisas de Marketing, Opinião e Mídia, 15(116), 61.

Kaplan, R. S., \& Norton, D. P. (2005). The balanced scorecard: measures that drive performance. Harvard

Business Review, 83(7), 172.

Kautz, K. (2009). Customer and user involvement in agile software development. The International Conference on Extreme

Programming and Agile Processes in Software Engineering, Oulu, Finlâdia.

Kirca, A. H., Bearden, W. O., \& Hult, G. T. M. (2011). Forms of market orientation and firm performance: A complementary approach. AMS Review, 1(3-4), 145-153.

Kirca, A. H., Jayachandran, S., \& Bearden, W. O. (2005). Market orientation: A meta-analytic review and assessment of its antecedents and impact on performance. Journal of Marketing, 69(2), 24-41.

Lee, J.-C., \& Chen, C.-Y. (2019). Investigating the environmental antecedents of organizations' intention to adopt agile software development. Journal of Enterprise Information Management, 32(35).

Liang, T.-P., \& Tanniru, M. (2006). Customer-centric information systems. Journal of Management Information Systems, 23(3), 9-15.

Lindvall, M., Muthig, D., Dagnino, A., Wallin, C., Stupperich, M., Kiefer, D., May, J., \& Kahkonen, T. (2004). Agile software development in large organizations. Computer, 37(12), 26-34.

Lishner, I., \& Shtub, A. (2019). Measuring the success of Lean and Agile projects: Are cost, time, scope and quality equally important? The 
Journal of Modern Project

Management, 7(1).

Loch, C., \& Sommer, S. (2019). The

Tension Between Flexible Goals

and Managerial Control in

Exploratory Projects. Project

Management Journal, 50(5), 524-

537.

Lohan, G., Conboy, K., \& Lang, M. (2011). Examining customer focus in IT project management: findings from Irish and Norwegian case studies. Scandinavian Journal of Information Systems, 23(2), 29-58.

Maiden, N., \& Jones, S. (2010). Agile Requirements Can We Have Our Cake and Eat It Too? IEEE Software, 27(3), 87-88.

Moe, N. B., Dingsøyr, T., \& Dybå, T. (2010). A teamwork model for understanding an agile team: A case study of a Scrum project. Information and Software Technology, 52(5), 480-491.

Moore, G. C., \& Benbasat, I. (1991). Development of an instrument to measure the perceptions of adopting an information technology innovation. Information Systems Research, 2(3), 192-222.

Nemoto, T., \& Beglar, D. (2014). Likertscale questionnaires. JALT 2013 Conference Proceedings, Tókio, Japão.

Ovaska, P., Rossi, M., \& Smolander, K. (2005). Filtering, negotiating and shifting in the understanding of information system requirements. Scandinavian Journal of Information Systems, 17(1), 7.
Parzinger, M. J., \& Nath, R. (2000). A study of the relationships between total quality management implementation factors and software quality. Total Quality Management, 11(3), 353-371.

Petersen, K. (2011). Measuring and predicting software productivity: A systematic map and review. Information and Software Technology, 53(4), 317-343.

Recker, J., Holten, R., Hummel, M., \& Rosenkranz, C. (2017). How agile practices impact customer responsiveness and development success: A field study. Project Management Journal, 48(2), 99121.

Serrador, P., \& Pinto, J. K. (2015). Does Agile work?-A quantitative analysis of agile project success. International Journal of Project Management, 33(5), 1040-1051.

Sheffield, J., \& Lemétayer, J. (2013). Factors associated with the software development agility of successful projects. International Journal of Project Management, 31(3), 459-472.

Siddique, L., \& Hussein, B. A. (2019). Enablers and barriers to customer involvement in agile software projects in Norwegian software industry: The Supplier's perspective. The Journal of Modern Project Management, 7(2).

Sousa, R. (2003). Linking quality management to manufacturing strategy: an empirical investigation 
of customer focus practices.

Journal of operations management, 21(1), 1-18.

Tenenhaus, M., Vinzi, V. E., Chatelin, Y.M., \& Lauro, C. (2005). PLS path modeling. Computational Statistics \& Data Analysis, 48(1), 159-205.

Werts, C. E., Linn, R. L., \& Jöreskog, K. G. (1974). Intraclass reliability estimates: Testing structural assumptions. Educational and Psychological Measurement, 34(1), 25-33.

Wetzels, M., Odekerken-Schröder, G., \& Van Oppen, C. (2009). Using PLS path modeling for assessing hierarchical construct models: Guidelines and empirical illustration. MIS Quarterly, 23(1), 177-195.

Wold, H. (1974). Causal flows with latent variables: partings of the ways in the light of NIPALS modelling. European Economic Review, 5(1), 67-86.

Wold, H. (1982). Soft modeling: the basic design and some extensions. Systems under Indirect Observation, 2, 343.

Yoshikuni, A. C., Galvão, F. R., \& Albertin, A. L. (2021). Knowledge strategy planning and information system strategies enable dynamic capabilities innovation capabilities impacting firm performance. VINE Journal of Information and Knowledge Management Systems.

Zultner, R. E. (1993). TQM for technical teams. Communications of the ACM, 36(10), 79-91. 